\title{
INDICADORES BIBLIOMÉTRICOS DOS ARTIGOS SOBRE HISTEROSCOPIA NA BASE SCOPUS
}

\author{
Denisley Henrique de JESUS, Marcela Tasca BARROS \& Eduardo SHIMODA \\ Universidade Candido Mendes. Campos dos Goytacazes, Rio de Janeiro, Brasil. \\ *Autor para correspondência: denisley@uol.com.br \\ DOI: http://dx.doi.org/10.18571/acbm.181
}

\section{RESUMO}

A histeroscopia é um método valioso no diagnóstico e/ou cirurgia de patologias endometriais. É de grande importância verificar a tendência de publicações a respeito do assunto e a bibliometria como ciência pode ajudar na avaliação dessa tendência. O objetivo do presente trabalho é apresentar indicadores bibliométricos a respeito do tema "histeroscopia", utilizando a base Scopus e comparando a evolução do Brasil e do mundo. Foi realizado uma pesquisa, na base Scopus, em julho de 2017 para verificação dos principais autores, instituições e periódicos que mais publicam a respeito do tema. Observa-se que o Brasil se encontra no $14^{\circ}$ lugar do ranking de publicações e as principais instituições que produziram artigos foram a USP, CEPARH e a UNIFESP. Conclui-se que o Brasil está bem atrás no número de publicações em comparação com principais países e que no ano de 2007, no Brasil, houve um grande aumento na produção desses artigos seguido de uma grande queda nos anos seguintes.

Palavras-chave: Histeroscopia; Bibliometria; Produção científica.

\begin{abstract}
Hysteroscopy is a valuable method in the diagnosis and / or surgery of endometrial pathologies. It is of great importance to check the tendency of publications on the subject and bibliometrics as science can help in the evaluation of this tendency. The aim of the present work is to present bibliometric indicators on the subject "hysteroscopy", using the Scopus base and comparing the evolution of Brazil and the world. A survey was carried out at the Scopus base in July 2017 to verify the main authors, institutions and periodicals that publish the most on the subject. It is observed that Brazil is in 14th place in the ranking of publications and the main institutions that produced articles were USP, CEPARH and UNIFESP. It is concluded that Brazil is far behind in the number of publications compared to the main countries and that in 2007, in Brazil, there was a great increase in the production of these articles followed by a great fall in the following years.
\end{abstract}

Key words: Hysteroscopy; Bibliometrics; Scientific production.

\section{Introdução}

Atualmente a histeroscopia é o método "padrão-ouro" no estudo da cavidade endometrial e cervical. Sua história de evolução se dá ao longo de cerca de 200 anos. Com o avanço tecnológico a medicina também obteve avanços nos procedimentos minimamente invasivos em algumas patologias ginecológicas. Os vídeos-histeroscópios atuais têm modernas tecnologias ópticas com melhor captação de imagens e calibre ultrafino que traz inúmeros benefícios no diagnóstico e tratamento das patologias endocervicais e endometriais, com maior eficácia e acurácia, possibilitando as mulheres um mais rápido retorno aos seus afazeres diários e diminuindo o tempo de permanência hospitalar. A importância de verificar tendências de publicações é evidente e a 


\section{Biomedica Brasiliensia}

ISSN: 2236-0867

bibliometria como ciência pode ajudar na avaliação sobre a dinâmica e evolução científica e tecnológica (SILVA FILHO et al., 2012).

O objetivo do presente trabalho foi realizar um estudo bibliométrico, usando a base de dados "Scopus", a respeito do tema "histeroscopia, possibilitando assim avaliar a produção científica nacional e internacional no tema proposto, sendo os resultados apresentados estratificados, indicando os índices e as tendências de publicação na área científica.

\section{Revisão de Literatura}

\subsection{Histeroscopia}

Nos dias de hoje a endoscopia é uma técnica usada em muitas especialidades médicas. Sendo um procedimento cirúrgico ou diagnóstico, a endoscopia é considerada um procedimento minimamente invasivo, com menos riscos e recuperação mais rápida para a paciente. A histeroscopia é a endoscopia da cavidade uterina, sendo um dos primeiros métodos desenvolvidos para visualização e estudo direto da cavidade uterina. A introdução da histeroscopia na prática ginecológica revolucionou o diagnóstico e o tratamento da doença intra-uterina. Novos desenvolvimentos metodológicos e tecnológicos tornaram a histeroscopia diagnóstica e operativa muito mais eficiente, econômica, segura e útil. A indicação mais comum de histeroscopia é o sangramento uterino anormal, mas também é usado em casos de infertilidade e anomalias Mullerianas (FILOGÔNIO et al., 2010).

Durante muito tempo, a curetagem foi considerada o padrão-ouro para investigações de lesões endometriais, embora um certo número de lesões focais (pólipos, hiperplasias, hiperplasias atípicas, cânceres) na cavidade uterina tenham sido perdidas (GIUSA- CHIFERI et al., 1996). Bettochi et al. relatou que as mulheres submetidas a curetagem não diagnosticavam $62,5 \%$ das principais desordens intrauterinas e os distúrbios endometriais estavam presentes no útero removido (BETTOCHI et al., 2001). Como é um procedimento às cegas, ele está associado a falhas diagnósticas que variam de 2-6\% (LOVERRO et al., 1999).

A histeroscopia provavelmente se tornará o novo padrão-ouro devido a sua capacidade de visualizar diretamente o endométrio e realizar biópsias direcionadas conforme indicado, permitindo a eliminação dos resultados falso-negativos da biópsia às cegas (LEE et al., 2008; ANGIONI et al., 2008). A histeroscopia tem a capacidade de reduzir os erros de amostragem e demonstrou um grande valor no planejamento do manejo terapêutico adequado para a patologia intracavitária e na detecção de patologias malignas (TINELLI et al., 2008).

A primeira histeroscopia foi realizada em 1986 e hoje é um método tanto para diagnóstico como para tratamento cirúrgico e vem sendo aperfeiçoado continuamente. É impressionante o avanço tecnológico que proporcionou a redução do diâmetro da óptica, segurança na distensão da cavidade uterina e transporte da luz pela fibra óptica. Em 1985, Bumm introduziu um cistoscópio para avaliar a cavidade uterina, realizando o diagnóstico e cauterizando endometrites, pólipos e miomas. Porém, somente em 1979 Hamou inventou a moderna histeroscopia, diminuindo o diâmetro da óptica, o que facilitou a progressão da mesma pelo canal cervical sem necessidade de dilatação e com uma imagem de qualidade. No Brasil, no final dos anos 80 é que chega a histeroscopia. Desde então a histeroscopia abriu novas possibilidades diagnósticas para avaliação do canal cervical e da cavidade uterina. Inovações técnicas recentes revolucionaram essa técnica. Tornou-se possível a realização de um exame endoscópico no próprio consultório, sem anestesia ou dilatação do canal cervical. A histeroscopia é o primeiro método desenvolvido para visualização e estudo direto da cavidade uterina. Este exame ainda auxilia na visualização direta do canal cervical, o que aumenta suas indicações e o torna um "padrão-ouro" na ginecologia, seja como diagnóstica ou cirúrgica (MENCAGLIA e ALBUQUERQUE NETO, 2002). A indicação mais comum da histeroscopia é o sangramento uterino anormal (SUA), mas também é indicada nos 
casos de infertilidade e malformações Mullerianas. O exame histeróscopico pode ser considerado o padrão-ouro para excluir patologias endometriais (FILOGÔNIO et al., 2010).

Com o avanço das técnicas e materiais endocópicos, na histeroscopia diagnóstica ambulatorial pode se realizar biópsia dirigida e exérese de pequenos pólipos, diminuindo o risco de complicacões, evitando-se procedimentos cirúrgicos maiores e até mesmo diminuir o tempo entre o diagnóstico e início do tratamento de lesões mais graves como hiperplasia complexas ou carcinoma de endométrio. As contra-indicações a realização da histeroscopia diagnóstica são poucas, o que já é uma vantagem do método. As contra-indicações absolutas são: gestação, infecção genital e metrorragia (SILVA FILHO et al., 2012). O exame de histerocopia, com ou sem amostragem endometrial, pode ser considerado o padrão-ouro de diagnóstico para excluir a patologia endometrial (FILOGÔNIO et al., 2010).

A histeroscopia cirúrgica é realizada no bloco cirúrgico sob analgesia, com o ressectoscópio, seja ele bipolar ou monopolar, com o objetivo de tratar lesões intracavitárias diagnósticadas previamente, como pólipos ou miomas submucosos. É o procedimento de escolha no tratamento de pólipos endometriais. Vários estudos demonstram que o risco de hiperplasia atípica e de câncer de endométrio associado a pólipos endométriais é cerca de $4 \%$, sendo aconselhável polipectomia em todos os casos diagnosticados. No tratamento de miomas submucosos, a histeroscopia cirúrgica é o tratamento de escolha, sendo geralmente indicada miomectomia histeroscópica em miomas submucosos menores que $4 \mathrm{~cm}$, para tratamento de menorragia ou de infertilidade. Aderências uterinas e septo uterino também apresentam como tratamento de escolha a histeroscopia cirúrgica. Com este procedimento cirúrgico minimamente invasivo pode-se tratar definitivamente patologias localizadas dentro da cavidade uterina, evitando-se cirurgias maiores e suas complicações (SILVA FILHO et al., 2012).

Aprender quando a visão histeroscópica pode excluir a existência de doença intra-uterina e determinar quais lesões apresentam maior probabilidade de ser confirmada pela histologia ainda representa um desafio, embora alguns estudos tenham sido publicados sobre a precisão da visão histeroscópica no diagnóstico da doença do endométrio. (GARUTI et al., 2001; CLARK et al., 2002). Melhor conhecimento da relação entre a imagem histeroscópica e a histologia endometrial ainda é necessária para melhorar sua precisão. Além disso, a maioria dos estudos publicados usam estimativas de sensibilidade e especificidade, bem como valores preditores, o que, infelizmente, nos fornece poucas informações sobre a probabilidade de um teste de diagnóstico ser preciso (ALTMAN e MATIN-BLAND, 1994).

Embora a histeroscopia tenha sido usada por muitos anos no diagnóstico de patologia intrauterina, sua sensibilidade e especificidade ainda são debatidas na literatura (GARUTI et al., 2001; CLARK et al., 2002; ALTMAN e MATIN-BLAND, 1994). O uso de diferentes sistemas de classificação para visão histeroscópica e histologia endometrial, o pequeno número de pacientes e a heterogeneidade em estudos publicados dificultam a estimativa da precisão histeroscópica (GARUTI et al., 2001; CLARK et al., 2002).

O acordo entre a visão histeroscópica e a histologia foi substancial para pólipos endometriais, miomas e cânceres de endométrio e moderado para hiperplasia endometrial simples. A avaliação histopatológica não pode ser omitida na presença de qualquer lesão, uma vez que a visão histeroscópica não pode substituir completamente o estudo histopatológico com anormalidades endometriais, especialmente no caso de hiperplasia endometrial. O estudo enfatiza a importância de correlacionar os achados de histerocopia com histopatologia do endométrio e o uso de estatísticas apropriadas para avaliarem a precisão do teste de diagnóstico (FILOGÔNIO et al., 2010).

A varredura de ultrassonografia transvaginal permite a detecção de patologia endometrial com alta sensibilidade para que possa ser utilizada como o primeiro passo de diagnóstico na triagem de mulheres pós-menopáusicas sintomáticas, mas não pode excluir completamente uma patologia endometrial. Deve ser completada pela avaliação histeroscópica quando a espessura do 
endométrio está alterada ou atrófica, para detectar o câncer e seus precursores em um estágio inicial (MARELLO e BETTOCHI, et al., 2000).

As mulheres com sangramento uterino que aparece pelo menos 12 meses ou mais após a menopausa precisam de diagnóstico correto antes de qualquer tratamento. Em aproximadamente $60 \%$ das mulheres com sangramento pós-menopausa nenhuma causa patológica no trato genital é identificada. A amostragem do endométrio mostra um endométrio atrófico e raramente proliferativo (CHOO et al., 1985). Os cânceres endometriais são associados com hemorragia uterina pós-menopausa como sintoma de apresentação em 90-95\% dos casos (FIORICA et al., 1990; GIUSA-CHIFERI et al., 1996). A amostragem endometrial sob anestesia geral, que obtém diretamente uma amostra de endométrio, ainda é o padrão-ouro para o estabelecimento de um diagnóstico (SEAMARK, 1998).

Alguns autores relatam que a curetagem da cavidade uterina tem uma taxa falso negativa entre $2 \%$ e $10 \%$ em casos de lesão focal (BOSSUYT et al.,2003). Todas as mulheres com hemorragia pós-menopausa devem ser submetidas imediatamente a um exame clínico e exame de ultrassonografia transvaginal (EPSTEIN e VALENTIN, 2004). A medida ultrassonográfica da espessura endometrial exibe uma boa precisão na distinção entre endométrio normal e patológico e contribui para a redução de exames invasivos desnecessários em mulheres pós-menopáusicas (AUSLENDER et al., 1993; GRANBERG et al.,1991).

A histeroscopia é uma técnica mais precisa que a ultrassonografia transvaginal por causa de uma melhor especificidade e deve ser indicada para todos as pacientes que apresentam faixa endometrial maior que 4mm de espessura (GARUTI et al.,1999). Este procedimento permite a visualização direta do endométrio e é superior a curetagem em fazer um diagnóstico preciso de patologias na cavidade uterina (GIMPELSON e RAPPOLD, 1988; LOFFER,1989). Comparado com outros métodos de exames não invasivos ou invasivos, a histeroscopia é de particular valor no diagnóstico de doenças benignas como pólipos endometriais e fibróides e miomas submucosos (BOURNE, 1995). Os casos de imagens histeroscópicas oncologicamente suspeitas devem ser sempre seguidos por amostragem endometrial e a remoção da lesão está indicada em todos as pacientes sintomáticas, bem como naquelas com risco definido para câncer de endométrio (FAMBRINI et al.,2008).

De acordo com relatos anteriores, a histeroscopia com biópsia endometrial é obrigatória nas mulheres pós-menopáusicas com sangramento uterino anormal (SUA) e pode ser usada de forma satisfatória como uma investigação de segunda linha para encontrar os motivos do sangramento (LITTA et al., 2005; TAHIR et al., 1999).

Percebe-se então, que a histeroscopia tem uma parte importante na avaliação do SUA em mulheres na pós-menopausa, desde que o sangramento uterino não seja um obstáculo definitivo para a visualização da cavidade endometrial, e esta seja suficientemente visualizada. Ao associar biópsia e visualização direta da cavidade uterina, a causa do sangramento pós-menopausa pode ser identificada com alta probabilidade. Outros projetos de pesquisa histeroscópica devem ser realizados no futuro em termos de avaliação da histeroscopia como ferramenta investigativa no manejo da hemorragia pós-menopausa (LIBERIS et al., 2010).

\subsection{Bibliometria}

A bibliometria é uma ferramenta estatística que permite mapear e gerar diferentes indicadores de tratamento e gestão da informação e do conhecimento, especialmente em sistemas de informação e de comunicação científicos e tecnológicos, e de produtividade, necessários ao planejamento, avaliação e gestão da ciência e tecnologia, de uma determinada comunidade científica ou país (GUEDES e BORSCHIVER, 2005).

Há, por parte de autores como Oliveira et al. (1992), a ideia de que a avaliação da produtividade científica, por exemplo, deve ser um dos elementos principais para o 
estabelecimento e acompanhamento de uma política nacional de ensino e pesquisa, uma vez que permite um diagnóstico das reais potencialidades de determinados grupos e/ou instituições.

A biliometria também se dedica ao estudo de artigos de periódicos, produtividade dos autores e estudo de citações. $\mathrm{O}$ estudo das citações permite estudar padrões na produção do conhecimento científico, tais como: autores mais citados, autores mais produtivos, procedência geográfica, procedência institucional dos autores mais influentes, tipo de publicação mais utilizado, a procedência da bibliografia utilizada, idade média e obsolência da literatura e periódicos mais citados. Sobre o uso de citações, alguns autores definem que a análise das citações é uma forma de se deduzir o impacto das publicações científicas, de forma a identificar autores mais produtivos e que contribuem efetivamente com a evolução da produção científica (ALVARADO, 1984; ALVARENGA, 1996).

O uso de técnicas bibliométricas contribui de forma decisiva em épocas de recursos escassos, quando um bibliotecário deve resolver que títulos ou publicações periódicas podem ou não ser suprimidas de uma biblioteca. Indicadores de uso são obtidos para definir uma lista de publicações periódicas prioritárias e para prever a demanda futura. É fundamental ter como detectar a utilização real dos títulos que constam em uma biblioteca, possibilitando determinar quais coleções estão ultrapassadas (RIVAS, 1981). Os índices bibliométricos também são utilizados para avaliar a produtividade e a qualidade da pesquisa dos cientistas, por meio da medição com base nos números de publicações e citações dos diversos pesquisadores (MEIS,1999).

Já para Araújo (2006), o ponto central da bibliometria é a utilização de métodos quantitativos, na busca por uma avaliação objetiva da produção científica. Para Bufrem e Prates (2005), é possível analisar, por meio dos indicadores bibliométricos, o desenvolvimento de um campo da ciência, a fim de identificar algumas características, como:

a) o crescimento cronológico da produção científica;

b) a produtividade de autores e instituições;

c) a colaboração entre pesquisadores e instituições;

d) o impacto das publicações;

e) a análise e avaliação de fontes difusoras de trabalhos e

f) a dispersão da produção científica entre diversas fontes.

O mapeamento apresentado por meio de estudos bibliométricos promove o conhecimento sobre o histórico e o estado atual do campo de pesquisa e disponibiliza insumos que permitem enriquecer a discussão sobre os possíveis rumos que as pesquisas na área têm tomado e as prováveis tendências científicas para os pesquisadores e interessados no tema (WOSZEZENKI e GONÇALVES, 2013).

\subsection{Base Scopus}

A Scopus é a maior base de dados de resumos e citações de literatura por pares, atualizada diariamente, apresentando, assim, o panorama mais completo da produção mundial de pesquisa nas áreas de ciência, tecnologia, medicina, ciências sociais e artes e humanidades. Inclui mais de 64 milhôes de registros, mais de 131.000 livros e mais de 21.500 publicações revisadas por pares (ELSEVIER, 2018)

A base de dados Scopus indexa 14.000 títulos de periódicos de 4.000 editoras internacionais, com atualizações diárias. A base Scopus cobre as seguintes áreas do conhecimento: química, física, matemática, engenharia, ciências da saúde e vida, ciências sociais, psicologia, economia, biologia, agricultura, ciências ambientais e ciências gerais. Mais de $60 \%$ dos títulos são de outros países que não os Estados Unidos e $85 \%$ do conteúdo é indexado utilizando os vocabulários controlados para a definição das palavras-chave e/ou descritores (MESQUITA et al., 2006). 


\section{Biomedica Brasiliensia}

\section{Metodologia}

Este trabalho trata-se de um estudo bibliográfico, que faz uso de técnicas bibliométricas, as quais apresentam natureza quantitativa. A bibliometria, como área de estudo em ciência da informação, possui papel fundamental na análise da produção científica, com indicadores que retratam o grau de desenvolvimento de uma área do conhecimento (MACHADO, 2007). A bibliometria, é uma técnica permite analisar o desenvolvimento de um campo da ciência, visando identificar suas características (WOSZEZENK e GONÇALVES, 2013).

A coleta de dados foi realizada na base Scopus, disponível no Portal Periódicos da Capes, foi realizada no dia 04 de julho de 2017, sendo usados o termo "histeroscopia" (em inglês: hysteroscopy). Buscaram-se os artigos que contivessem este termo no título, resumo ou palavraschaves, limitando-se a busca àqueles artigos publicados em periódicos. Inicialmente foram obtidos os dados gerais e, posteriormente, restritos aos trabalhos de brasileiros. As expressões de busca com operadores booleanos foram (Quadro 1):

TITLE-ABS-KEY (hysteroscopy) AND (LIMIT-TO (DOCTYPE, "ar")) AND (LIMIT-TO (SRCTYPE, "j")): 6854

TITLE-ABS-KEY (hysteroscopy) AND (LIMIT-TO (DOCTYPE, "ar")) AND (LIMIT-TO (SRCTYPE, "j")) AND (LIMIT-TO (AFFILCOUNTRY, "Brazil")): 157

Quadro 1: Expressões de busca.

Obtiveram-se informações relacionadas à quantidade de artigos por ano, autor, área, instituição, país e periódico.

\section{Resultados e Discussão}

\subsection{Principais Países}

Nota-se na figura 1 que o país que mais publica a respeito do tema são os Estados Unidos (EUA), com 14,3\% das publicações, ficando logo abaixo dele a Itália e o Reino Unido, sendo que os Estados Unidos apresentam quase que 5 pontos percentuais a mais de publicações sobre o assunto que os outros países. Observa-se também, que o Brasil aparece em $14^{\circ}$ lugar, com apenas 2,3\% das publicações, possivelmente por ser a "histeroscopia" uma técnica de diagnóstico e tratamento endoscópico relativamente nova que foi primeiramente desenvolvida e aplicada nos países desenvolvidos para posteriormente ser utilizada nos países em desenvolvimento. Quando a histeroscopia começou a ser desenvolvida no Brasil, o material utilizado era todo proveniente dos Estados Unidos e Europa, sendo de difícil acesso pelo alto custo de importação e manutenção. Na atualidade, várias empresas brasileiras já fabricam tais produtos o que melhorou a acessibilidade e a prática da histeroscopia em todo Brasil. 


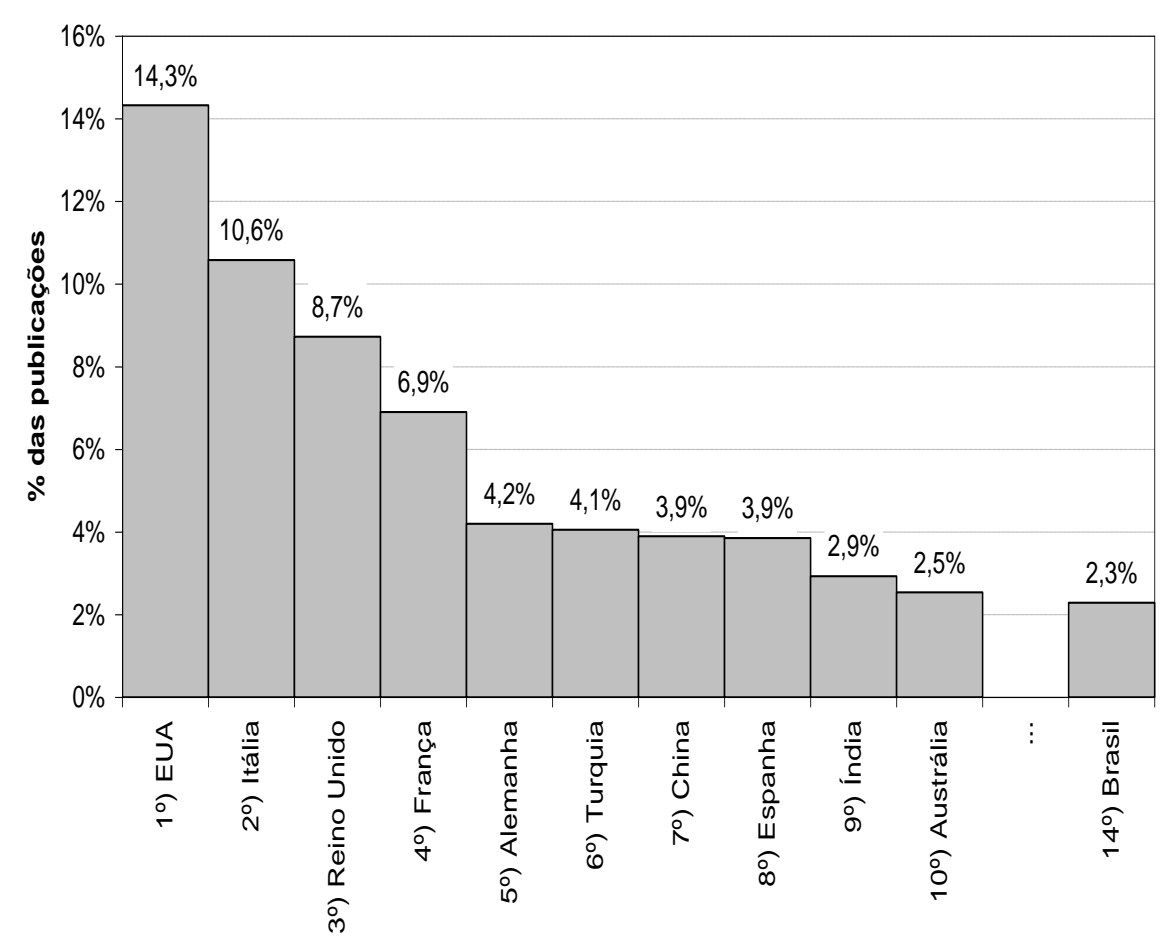

Figura 1: Países com mais publicações sobre o tema histeroscopia na base Scopus.

\subsection{Principais Instituições}

A figura 2 representa as principais instituições, nacionais e internacionais, com mais publicações a respeito de histeroscopia. No Brasil as instituições que mais publicam estão, a maioria, no estado de São Paulo e seguido do estado da /Bahia, Rio de Janeiro e Minas Gerais.

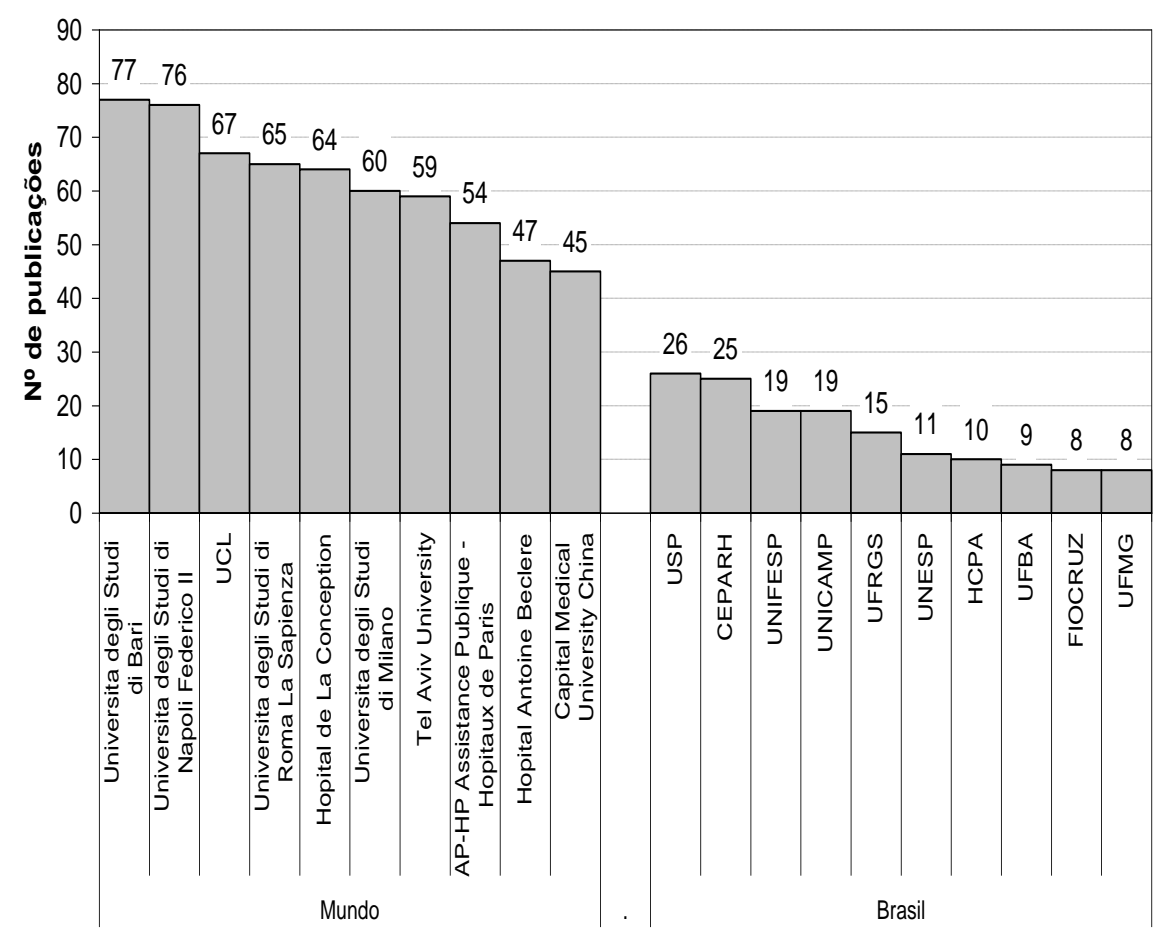




\section{Biomedica Brasiliensia}

ISSN: 2236-0867

Figura 2: Número de publicações das instituições que mais publicam no mundo e no Brasil. Abreviaturas: USP Universidade São Paulo; CEPARH - Centro de Pesquisa e Assistência em Reprodução Humana; UNIFESP Universidade Federal de São Paulo; UNICAMP - Universidade Estaudal de Campinas; UFRGS - Universidade Federal do Rio Grande do Sul; UNESP - Universidade Estaudal Paulista; HCPA - Hospital de Clínicas de Porto Alegre; UFBA - Universidade Federal da Bahia; FIOCRUZ - Fundação Oswaldo Cruz; UFMG - Universidade Federal de Minas Gerais.

\subsection{Evolução Temporal}

Quando se relaciona o número de publicações do Brasil e do mundo e o ano dessas, notase que no mundo a curva de publicações mantém-se numa constante e com um número mais elevado de publicações, tendo apresentado um aumento em 2007 e mantendo este aumento até 2015. O Brasil apresenta oscilações nessa curva, com um pico significante de publicações em 2007, ultrapassando as publicações do mundo, mesmo período em que a técnica de histeroscopia diagnóstica e cirúrgica se difundiu pelo Brasil, e apresenta uma queda considerável nas publicações a partir desse pico, sendo inverso o ocorrido no mundo.

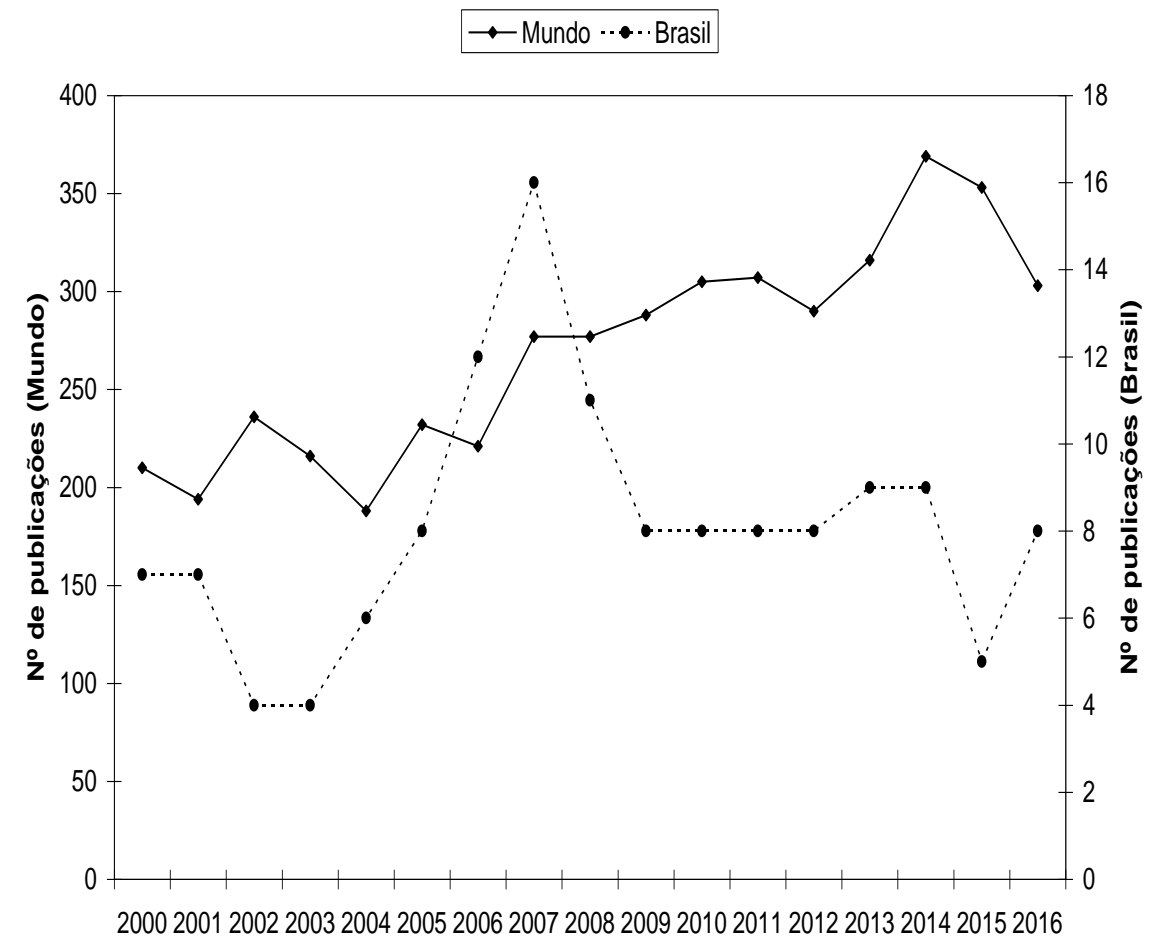

Figura 3: Número de publicações no Brasil e no mundo e ano dessas publicações.

\subsection{Principais Autores}

A figura 4 mostra os autores que mais publicaram no mundo e no Brasil separadamente, sendo o número de publicações no Brasil ainda muito inferior do restante do mundo. $\mathrm{O}$ autor no Brasil que mais publicou, ainda publicou um número inferior ao autor que menos publicou no mundo. 


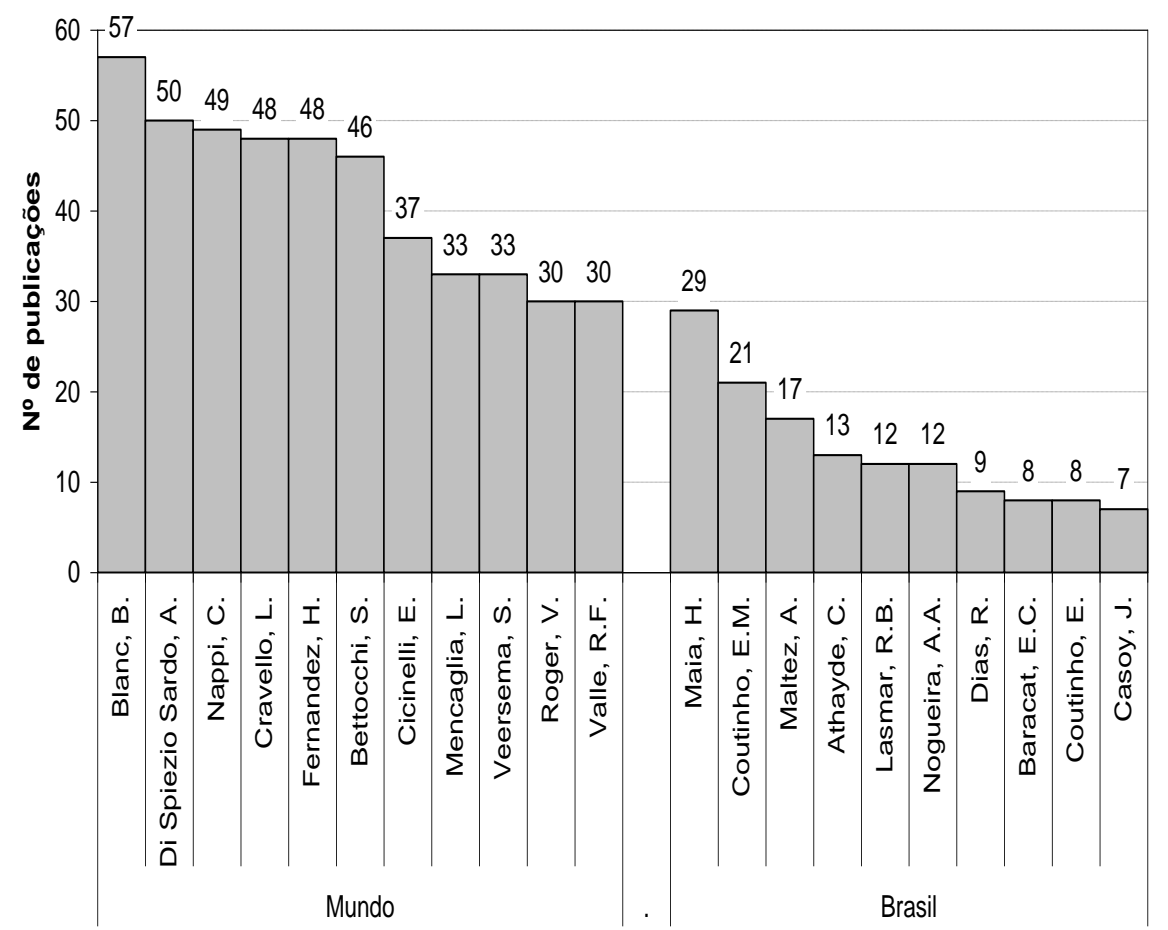

Figura 4: Autores com maiores publicações sobre histeroscopia no mundo e no Brasil.

\subsection{Principais Periódicos}

A figura 5 relaciona o nome das revistas que mais publicaram no Brasil e no mundo, comprovando, novamente, que o número de publicações do Brasil é muito inferior ao número de publicações do mundo.

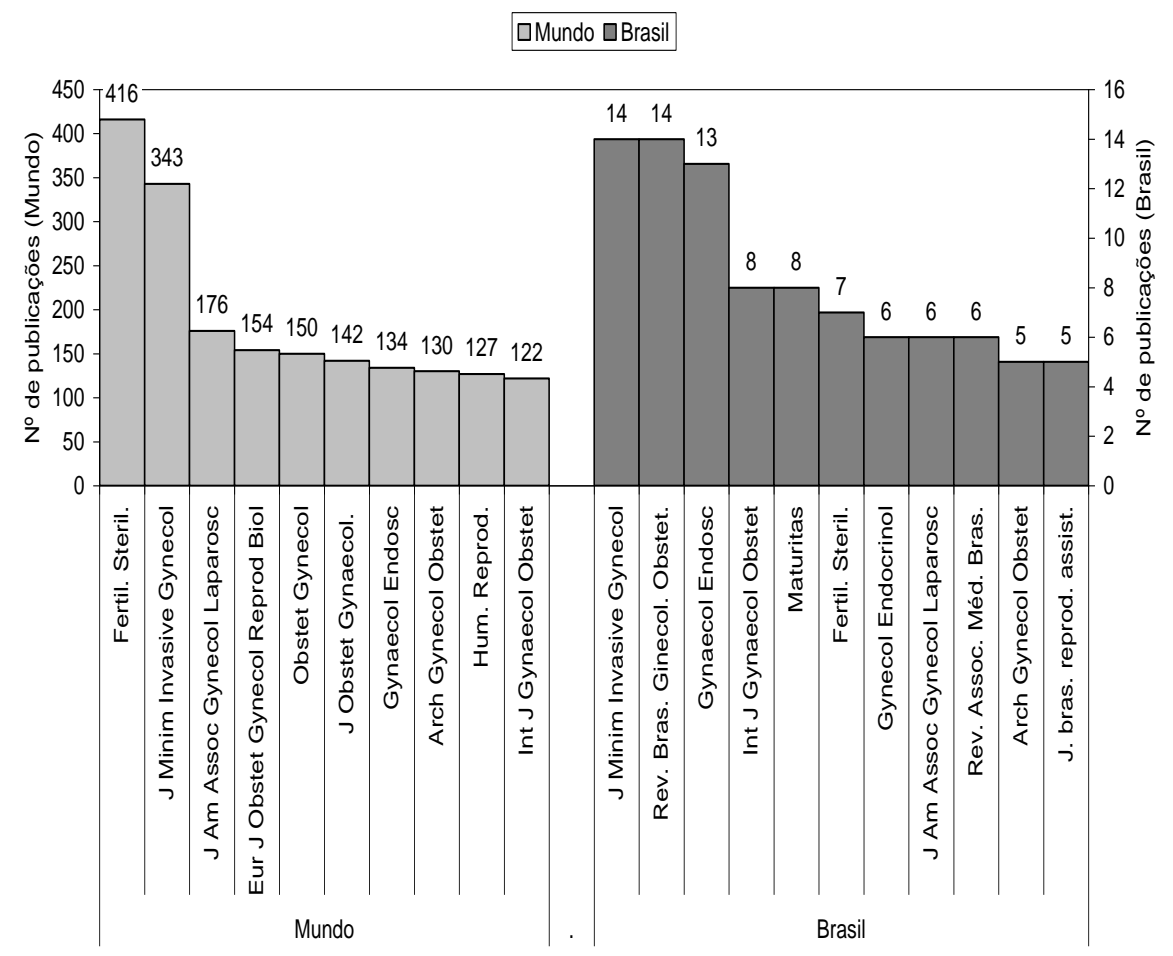


Figura 5: Representa os principais periódicos nos quais foram veiculados artigos a respeito do tema. Abreviaturas: Arch Gynecol Obstet (Archives Of Gynecology And Obstetrics); Eur J Obstet Gynecol Reprod Biol (European Journal Of Obstetrics Gynecology And Reproductive Biology); Fertil. Steril. (Fertility And Sterility); Gynaecol Endosc (Gynaecological Endoscopy); Gynecol Endocrinol (Gynecological Endocrinology); Hum. Reprod. (Human Reproduction); Int J Gynaecol Obstet (International Journal Of Gynecology And Obstetrics); J Am Assoc Gynecol Laparosc (Journal Of The American Association Of Gynecologic Laparoscopists); J Minim Invasive Gynecol (Journal Of Minimally Invasive Gynecology); J Obstet Gynaecol. (Journal Of Obstetrics And Gynaecology); Maturitas (Maturitas); Obstet Gynecol (Obstetrics And Gynecology); Rev. Assoc. Méd. Bras. (Revista Da Associacao Medica Brasileira); Rev. Bras. Ginecol. Obstet. (Revista Brasileira De Ginecologia E Obstetrícia)

\section{Considerações Finais}

Conclui-se que o Brasil está bem atrás no número de publicações em comparação com os principais países e que no ano de 2007, no Brasil, houve um grande aumento na produção desses artigos seguido de uma grande queda nos anos subsequentes e que ele se encontra em $14^{\circ}$ lugar no ranking de publicações sobre o tema. A bibliometria é importante para avaliarmos o quanto um tema tem sido estudado no Brasil e no mundo. A histeroscopia é um avanço tecnológico e científico, tanto para diagnóstico como para tratamento cirúrgico, imprecindível nos dias de hoje. Porém mais estudos precisam acontecer para ampliar o conhecimento nessa área

\section{Referências}

ALTMAN, D. G; BLAND, J M. Statistics Notes: Diagnostic tests 2. Bmj, [s.1.], v. 309, n. 6947, p. 102-102, 9 jul. 1994.

ALVARADO, Rubén Urbizagástegui. A bibliometria no Brasil. Ciência da Informação, Brasília, v. 13, n. 2, p. 91-105, 1994.

ALVARENGA, Lídia. A Institucionalização da Pesquisa Educacional no Brasil. 1996. 249 f. Tese (Doutorado) - Curso de Educação, Universidade Federal de Minas Gerais, Belo Horizonte, 1996.

ANGIONI, Stefano et al. Detection of Benign Intracavitary Lesions in Postmenopausal Women with Abnormal Uterine Bleeding: A Prospective Comparative Study on Outpatient Hysteroscopy and Blind Biopsy. Journal of Minimally Invasive Gynecology, [s.1.], v. 15, n. 1, p. 87-91, jan. 2008 .

AUSLENDER, R. et al. Vaginal ultrasonography in patients with postmenopausal bleeding: Sonography in postmenopausal bleeding. Ultrasound in Obstetrics and Gynecology, v. 3, n. 6, p. 426-428, 1 nov. 1993.

BETTOCCHI, S. Diagnostic inadequacy of dilatation and curettage. Fertility and Sterility, v. 75, n. 4, p. 803-805, abr. 2001.

BOSSUYT, P. M. et al. Towards Complete and Accurate Reporting of Studies of Diagnostic Accuracy: The STARD Initiative. Clinical Chemistry and Laboratory Medicine, v. 41, n. 1, 27 jan. 2003.

BOURNE, T. H. Evaluating the endometrium of postmenopausal women with transvaginal ultrasonography: Opinion. Ultrasound in Obstetrics and Gynecology, v. 6, n. 2, p. 75-80, 1 ago. 1995. 
BUFREM, L.; PRATES, Y. O saber científico registrado e as práticas de mensuração da informação. Ciência da Informação, Brasília, v. 34, n. 2, p. 9-25, 2005.

CHOO, Y. C. et al. Postmenopausal uterine bleeding of nonorganic cause. Obstetrics and Gynecology, v. 66, n. 2, p. 225-228, ago. 1985.

CLARK, T. J. et al. Accuracy of Hysteroscopy in the Diagnosis of Endometrial Cancer and Hyperplasia: A Systematic Quantitative Review. JAMA, v. 288, n. 13, p. 1610, 2 out. 2002.

EPSTEIN, E.; VALENTIN, L. Managing women with post-menopausal bleeding. Best Practice \& Research Clinical Obstetrics \& Gynaecology, v. 18, n. 1, p. 125-143, fev. 2004.

FAMBRINI, M. et al. Clinical utility of liquid-based cytology for the characterization and management of endometrial polyps in postmenopausal age. International Journal of Gynecological Cancer, v. 18, n. 2, p. 306-311, mar. 2008.

FILOGÔNIO, I. D. de S. et al. Accuracy of Hysteroscopic View in the Diagnosis of Intrauterine Pathology: A Brazilian Experience. Journal of Gynecologic Surgery, v. 26, n. 1, p. 23-30, mar. 2010.

FIORICA, J. V. et al. Detection of endometrial carcinoma: clinical judgement versus histologic examination. Southern Medical Journal, v. 83, n. 7, p. 759-760, jul. 1990.

GARUTI, G. et al. Hysteroscopy and transvaginal ultrasonography in postmenopausal women with uterine bleeding. International Journal of Gynecology \& Obstetrics, [s.1.], v. 65, n. 1, p. 25-33, abr. 1999.

GARUTI, G. et al. Accuracy of Hysteroscopy in Predicting Histopathology of Endometrium in 1500 Women. The Journal of the American Association of Gynecologic Laparoscopists, v. 8, n. 2, p. 207-213, maio 2001.

GIMPELSON, R. J.; RAPPOLD, H. O. A comparative study between panoramic hysteroscopy with directed biopsies and dilatation and curettage. American Journal of Obstetrics and Gynecology, v. 158, n. 3, p. 489-492, mar. 1988.

GIUSA-CHIFERI, M. G. et al. Transvaginal ultrasound, uterine biopsy and hysteroscopy for postmenopausal bleeding. International Journal of Gynecology \& Obstetrics, v. 55, n. 1, p. 3944, out. 1996.

GRANBERG, S. et al. Endometrial thickness as measured by endovaginal ultrasonography for identifying endometrial abnormality. American Journal of Obstetrics and Gynecology, v. 164, n. 1, p. 47-52, jan. 1991.

GUEDES, V. L. S.; BORSCHIVER, S. Bibliometria: uma ferramenta estatística para a gestão da informação e do conhecimento, em sistemas de informação, de comunicação e de avaliação científica e tecnológica. In: ENCONTRO NACIONAL DE CIÊNCIA DA INFORMAÇÃ̃O (CINFORM), 6, 2005, Salvador, Anais do VI Encontro Nacional de Ciências da Informação, Salvador, UFBA, 2005. 
LEE, C. L. et al. The Roles of Endoscopy in Endometrial Cancer. Taiwanese Journal of Obstetrics and Gynecology, v. 47, n. 4, p. 379-383, dez. 2008.

LIBERIS, V. et al. The contribution of hysteroscopy to the detection malignancy in symptomatic postmenopausal women. Minimally Invasive Therapy \& Allied Technologies, v. 19, n. 2, p. 8393, abr. 2010.

LITTA, P. et al. Role of hysteroscopy with endometrial biopsy to rule out endometrial cancer in postmenopausal women with abnormal uterine bleeding. Maturitas, v. 50, n. 2, p. 117-123, fev. 2005 .

LOFFER, F. D. Hysteroscopy with selective endometrial sampling compared with D\&C for abnormal uterine bleeding: the value of a negative hysteroscopic view. Obstetrics and Gynecology, v. 73, n. 1, p. 16-20, jan. 1989.

LOVERRO, G. et al. Transvaginal sonography and hysteroscopy in postmenopausal uterine bleeding. Maturitas, v. 33, n. 2, p. 139-144, out. 1999.

MACHADO, Raimundo das Neves. Análise cientométrica dos estudos bibliométricos publicados em periódicos da área de biblioteconomia e ciência da informação (1990-2005). Perspectivas em Ciência da Informação, Belo Horizonte, v. 12, n. 3, p. 2-20, 2007.

MARELLO, F. et al. Hysteroscopic evaluation of menopausal patients with sonographically atrophic endometrium. The Journal of the American Association of Gynecologic Laparoscopists, v. 7, n. 2, p. 197-200, maio 2000.

MEIS, L. et al. Uso de indicadores exige cautela. Folha de São Paulo, São Paulo, 12 set. 1999. Caderno Especial Ranking da Ciência, p. 7.

MENCAGLIA, L. et al. Histerosocopia Diagnóstica. Medsi, 2002. 243 p.

MESQUITA, R. et al. Elaboração e aplicação de instrumentos para avaliação da base de dados Scopus. Perspectivas em Ciência da Informação, v. 11, p. 187-205, 2006.

OLIVEIRA, A. C. et al. A Bibliometria na avaliação da produção científica da área de nutrição registrada no Cibran: período de 1984-1989. Ciência da Informação, Brasília, v. 21, n. 3, p. 239242, set./dez. 1992.

RIVAS, L. M. Técnicas bibliométricas: selección y evaluación de publicaciones periódicas para bibliotecas y bases de datos biomédicas especializadas. Bibliotecología y Documentación, v. 6, n. 6-11, p. 41-81, jul/dic. 1981.

SEAMARK C.J. Endometrial sampling in general practice. Br J gen Pract., v. 48, p. 1597-8, 1998. SILVA FILHO, A. L. da. et al. Manual de Ginecologia e Obstetrícia SOGIMG. 5. ed. Belo Horizonte: Coopmed, 2012.

TAHIR, M. M. et al. A randomised controlled trial comparing transvaginal ultrasound, outpatient hysteroscopy and endometrial biopsy with inpatient hysteroscopy and curettage. BJOG: An International Journal of Obstetrics and Gynaecology, v. 106, n. 12, p. 1259-1264, dez. 1999. 
TINELLI, R. et al. The role of hysteroscopy with eye-directed biopsy in postmenopausal women with uterine bleeding and endometrial atrophy: Menopause, v. 15, n. 4, p. 737-742, jul. 2008.

WOSZEZENKI, C. R.; GONÇALVES, A. L. Mineração de textos biomédicos: uma revisão bibliométrica. Perspectivas em Ciência da Informação, v. 18, n. 3, p. 24-44, jul./set. 2013. 